\title{
Congestion Control for Nonlinear TD-SCDMA Discrete Networks Based on TCP/IP
}

\author{
http://dx.doi.org/10.3991/ijoe.v11i7.4761 \\ Peng Liu \\ Chongqing College of Electronic Engineering, China
}

\begin{abstract}
A successive approximation approach (SAA) is developed to obtain a new congestion controller for the nonlinear TD-SCDMA network control systems based on TCP/IP. By using the successive approximation approach, the original optimal control problem is transformed into a sequence of nonhomogeneous linear two-point boundary value (TPBV) problems. The optimal control law obtained consists of an accurate linear feedback term and a nonlinear compensation term that is the limit of the solution sequence of the adjoint vector differential equations. By using the finite-time iteration of nonlinear compensation term of optimal solution sequence, we can obtain a suboptimal control law for TD-SCDMA network control systems based on TCP/IP.
\end{abstract}

Index Terms-TD-SCDMA, TCP/IP, successive approximation approach, congestion controller, network control systems, two-point boundary value problems.

\section{INTRODUCTION}

It is well known that the insertion of the TD-SCDMA network based on TCP/IP in the feedback control loop makes the analysis and design of a network control systems complex because the network imposes an undetermined communication delay ${ }^{[1]}$. Therefore, conventional control theories with many ideal assumptions must be revaluated before they can be applied to network control systems. For instance, the stochastic optimal controller and the optimal state estimator of a network control system whose network induced delay is shorter than a sampling period have been proposed by Nilsson ${ }^{[2]}$. In Ref. [3] a model-based network control system was introduced. This control architecture has as main objective the reduction of the data packets transmitted over the network by a networked control system.

TD-SCDMA Network control systems based on TCP/IP can be described by nonlinear systems ${ }^{[4]}$. An amount of literature related to the analysis and controller design of such systems has been developed over the past decades. The stability region estimation and controller design for nonlinear systems with uncertainties are considered $^{[5]}$. While for the quadratic cost functional in the state and control, the optimal state feedback control problem often leads to solving a Hamilton-Jacobi-Bellman (HJB) equation or a nonlinear two-point boundary value (TPBV) problem. But for the general regulation problem of nonlinear systems, with the exception of simplest case, there is no analytic optimal control in explicit feedback form. This has spirited up researchers to develop many methods to obtain an approximate solution to the HJB equations or the nonlinear TPBV problems as well as obtain a suboptimal feedback control ${ }^{[6-7]}$.

Since TD-SCDMA network control systems based on $\mathrm{TCP} / \mathrm{IP}$ are an integrated research area, which is not only concerned about control, but also relevant to communication, we must combine the knowledge of control and communication together to improve the system performance. Following this direction, in this paper, we address a novel scheme that integrates control technology with communication technology for a class of nonlinear network control systems ${ }^{[8]}$.

\section{PROBLEM FORMULATION}

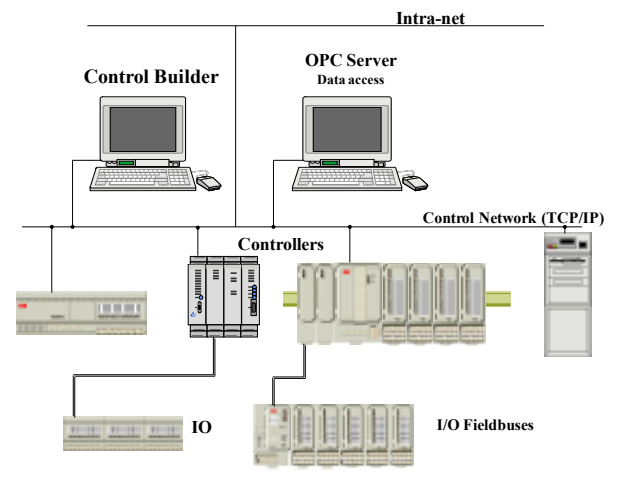

Figure 1. A collection of networked control systems shared by a communication link

We consider the TD-SCDMA networked control systems based on TCP/IP consisting of a collection of nonlinear plants whose feedback control loops are closed via a shared network link, as illustrated in Figure 1. All sample values of plant states are transmitted in one package ${ }^{[9]}$. The $k$-th plant is given by

$$
\left\{\begin{array}{l}
x(k+1)=g(x(k))+B u(k) \\
x(0)=x_{0}
\end{array}\right.
$$

where $x$ is an $n$-dimensional real state vector, $u$ an $r$ dimensional real control vector, $B$ an $n \times r$ constant matrix, $x_{0}$ a known initial state vector. Assume that $g \in C^{\infty}\left(R^{n} \rightarrow U \subset R^{n}\right), g(0)=0$.

Nonlinear function sequence $g$ may be expanded into the series form

$$
g(x(k))=A x(k)+f(x(k))
$$


where $f$ is the nonlinear term whose order size is larger than 1 with respect to $x, A=\frac{\partial g(0)}{\partial x^{T}}$. Therefore, system (1) may be rewritten as

$$
\left\{\begin{array}{l}
x(k+1)=A x(k)+f(x(k))+B u(k) \\
x(0)=x_{0}
\end{array}\right.
$$

The control objective, in an optimal control sense, is to find a control law $u^{*}(k)$, which may make the quadratic performance index

$$
J=\frac{1}{2} \sum_{k=0}^{N-1}\left[x^{T}(k) Q x(k)+u^{T}(k) R u(k)\right]
$$

where $R$ is an $r \times r$ positive-definite matrix and $Q_{f}, Q$ are $n \times n$ semi-positive-definite matrices.

\section{PRELIMINARIES}

As we know, we may get the optimal control law of the quadratic performance index (4) if and only if the system in (1) satisfies the following two-point boundary value problem:

$$
\left\{\begin{array}{l}
\lambda(k)=Q x(k)+f_{x}^{T}(x(k)) \lambda(k+1) \\
x(k+1)=A x(k)+B u(k)+f(x(k)), \\
u(k)=-R^{-1} B^{T} \lambda(k+1) \\
\quad \text { with the boundary conditions: }
\end{array}\right.
$$

$$
\left\{\begin{array}{l}
x(0)=x_{0} \\
\lambda(N)=Q_{f} x(N)
\end{array}\right.
$$

where $f_{x}^{T}=\frac{\partial f^{T}}{\partial x}$. Since (5) is a nonlinear two-point boundary problem, in a general way, it is difficult to get the solution whether the exact solution or the numerical solution.

We will propose a sensitivity approach to simplify the two-point boundary value problem in (5) and help get the optimal control law. Construct the following twopoint boundary value problem, in which a sensitive parameter $\varepsilon$ is introduced

$$
\left\{\begin{aligned}
\lambda(k, \varepsilon)= & Q x(k, \varepsilon)+A^{T} \lambda(k+1, \varepsilon) \\
& +\varepsilon f_{x}^{T}(x(k), \varepsilon) \lambda(k+1, \varepsilon) \\
x(k+1, \varepsilon) & =A x(k, \varepsilon)+B u(k, \varepsilon) \\
& +\varepsilon f(x(k), \varepsilon),
\end{aligned}\right.
$$

with boundary conditions

$$
\left\{\begin{array}{l}
x(0, \varepsilon)=x_{0} \\
\lambda(N, \varepsilon)=Q_{f} x(N)
\end{array}\right.
$$

where $0 \leq \varepsilon \leq 1$. Obviously, when $\varepsilon=1$, the two-point boundary value problem in (6) is equivalent to the original problem in (5).

\section{OPTIMAL CONTROL DESIGN}

In the following research, assume that $u(k, \varepsilon), x(k, \varepsilon)$ and $\lambda(k, \varepsilon)$ are infinite differentiable with respect to $\varepsilon$ around $\varepsilon=0$. Expending Maclaurin series, then

$$
\begin{aligned}
u(k, \varepsilon) & =\sum_{i=0}^{\infty} \frac{\varepsilon^{i}}{i !} u^{(i)}(k) \\
x(k, \varepsilon) & =\sum_{i=0}^{\infty} \frac{\varepsilon^{i}}{i !} x^{(i)}(k), \\
\lambda(k, \varepsilon) & =\sum_{i=0}^{\infty} \frac{\varepsilon^{i}}{i !} \lambda^{(i)}(k)
\end{aligned}
$$

where superscript $(i)$ denotes the $i$ th-order derivative of the series with respect to $\varepsilon$ when $\varepsilon=0$.

In order to guarantee convergence for the series in (7) when $\varepsilon=1$, we give the following result.

Theorem 1 There exist proper function sequences $u(k, \varepsilon), x(k, \varepsilon)$ and $\lambda(k, \varepsilon)$ with respect to $\varepsilon$, such that the series in (7) are convergent to $u(k), x(k)$, and $\lambda(k)$ when $\varepsilon=1$ respectively.

Proof Define $x(k, \varepsilon)=x(k) e^{\varepsilon-1}, u(k, \varepsilon)=u(k) e^{\varepsilon-1}$ and $\lambda(k, \varepsilon)=\lambda(k) e^{\varepsilon-1}$. For simplicity and without loss the generality, we only discuss the convergence for the series of $x(k, \varepsilon)$ in (7) when $\varepsilon=1$. Expending the Maclaurin series, we may get

$$
x(k, \varepsilon)=e^{-1}\left(\sum_{i=0}^{\infty} \frac{\varepsilon^{i}}{i !}\right) x(k)
$$

When $\varepsilon=1$, then

$$
x(k, 1)=e^{-1}\left(\sum_{i=0}^{\infty} \frac{1}{i !}\right) x(k)=x(k) .
$$

According to the same reasoning process, we may also get the conclusion that

$$
\begin{aligned}
& u(k, 1)=e^{-1}\left(\sum_{i=0}^{\infty} \frac{1}{i !}\right) u(k)=u(k), \\
& \lambda(k, 1)=e^{-1}\left(\sum_{i=0}^{\infty} \frac{1}{i !}\right) \lambda(k)=\lambda(k)
\end{aligned}
$$

The theorem 1 shows that the two-point boundary problem in (6) with the sensitivity parameter $\varepsilon$ is equal to problem (5) when $\varepsilon=1$.

Note that the Maclaurin series of $\varepsilon f(x(k), \varepsilon)$ is expanded as following

$$
\varepsilon f(x(k), \varepsilon)=\sum_{i=0}^{\infty} \frac{i f\left(x^{(i-1)}(k)\right)}{i !} \varepsilon^{i}
$$

and $\varepsilon f_{x}^{T}(x(k), \varepsilon) \lambda(k+1, \varepsilon)$ is expanded as 


$$
\begin{aligned}
& \varepsilon f_{x}^{T}(x(k), \varepsilon) \lambda(k+1, \varepsilon)= \\
& \sum_{i=0}^{\infty} \frac{i \sum_{j=0}^{i-1} C_{i-1}^{j} f_{x}^{T}\left(x^{(j)}(k)\right) \lambda^{(i-1-j)}(k+1)}{i !} \varepsilon^{i}
\end{aligned}
$$

where

$$
\lambda^{(-1)}(k)=x^{(-1)}(k)=0, \quad C_{i}^{j}=\frac{i !}{j !(i-j) !}
$$

Substituting (7), (11), and (12) into the two sides of (6), we may obtain

$$
\left\{\begin{array}{l}
x^{(i)}(k+1)=A x^{(i)}(k)+i f\left(x^{(i-1)}(k)\right)+B u^{(i)}(k), \\
\lambda^{(i)}(k)=i \sum_{j=0}^{i-1} C_{i-1}^{j} f_{x}^{T}\left(x^{(j)}(k)\right) \lambda^{(i-1-j)}(k+1)
\end{array}\right.
$$

with boundary conditions

$$
\left\{\begin{array} { l } 
{ x ^ { ( 0 ) } ( 0 ) = x _ { 0 } } \\
{ \lambda ^ { ( 0 ) } ( N ) = Q _ { f } x ( N ) }
\end{array} \quad \left\{\begin{array}{l}
x^{(i)}(0)=0 \\
\lambda^{(i)}(N)=0
\end{array}\right.\right.
$$

When $i=0$, we get the 0th-order two-point boundary problem

$$
\left\{\begin{array}{l}
x^{(0)}(k+1)=A x^{(0)}(k)+B u^{(0)}(k), \\
\lambda^{(0)}(k)=Q x^{(0)}(k)+A^{T} \lambda^{(0)}(k+1)
\end{array}\right.
$$

with boundary conditions

$$
\left\{\begin{array}{l}
x^{(0)}(0)=x_{0} \\
\lambda^{(0)}(N)=Q_{f} x(N)
\end{array}\right.
$$

Let

$$
\lambda^{(0)}(k)=P(k) x^{(0)}(k)
$$

Substituting (17) into (16), we obtain the 0th-order optimal control law as follows:

$$
u^{(0)}(k)=-S^{-1}(k+1) B^{T} P(k+1) A x^{(0)}(k)(18)
$$

Where $S(k)=R+B^{T} P(k) B$, and $P(k)$ is the positive-definite solution of the following Riccati matrix difference equation

$$
\left\{\begin{array}{l}
P(k)=A^{T}\left[I-P(k+1) B S^{-1}(k+1) B^{T}\right] \\
P(N)=Q_{f}
\end{array}\right.
$$

Noting that if $\left(x^{(i-1)}(k)\right)$ and

$$
i \sum_{j=0}^{i-1} C_{i-1}^{j} f_{x}^{T}\left(x^{(j)}(k)\right) \lambda^{(i-1-j)}(k+1)
$$

are known functions which are the solutions obtained in the $(i-1)$ th iteration, two-point boundary problem in (20) is a linear nonhomogeneous one. In order to solve this problem, let

$$
\lambda^{(i)}(k)=P(k) x^{(i)}(k)+g_{i}(k)
$$

Substituting (21) into (20), we may decouple the twopoint boundary problem (20), and get the $i$ th costate equation

$$
\left\{\begin{array}{l}
g_{i}(k)=A^{T}\left[I-P(k+1) B S^{-1}(k+1) B^{T}\right] \\
+i \sum_{j=0}^{i-1} C_{i-1}^{j} f_{x}^{T}\left(x^{(j)}(k)\right) \lambda^{(i-1-j)}(k+1) \\
g_{i}(N)=0 \quad k=0,1,2, \cdots
\end{array}\right.
$$

\section{AN ILLUSTRATED EXAMPLE}

Consider the optimal control problem for a bilinear model of a TD-SCDMA networked control system based on TCP/IP described by (1) and (3), where

$$
\begin{aligned}
& A=\left[\begin{array}{cc}
11 / 6 & 5 / 12 \\
-40 / 3 & -8 / 3
\end{array}\right], \quad B=\left[\begin{array}{c}
-1 / 8 \\
0
\end{array}\right], \\
& N_{1}=\left[\begin{array}{c}
-1 \\
0
\end{array}\right], \quad N_{2}=\left[\begin{array}{l}
0 \\
0
\end{array}\right], \quad\left[\begin{array}{l}
x_{1}(0) \\
x_{2}(0)
\end{array}\right]=\left[\begin{array}{c}
0.1 \\
0
\end{array}\right] \\
& Q_{f}=\left[\begin{array}{cc}
1020 & 0 \\
0 & 1020
\end{array}\right], \quad Q=\left[\begin{array}{cc}
10 & 0 \\
0 & 10
\end{array}\right], \\
& R=1, \quad t_{0}=0, \quad t_{f}=3
\end{aligned}
$$

The state variables $x_{1}$ and $x_{2}$ represent temperature and concentration of the initial product of the chemical reaction, respectively. The scalar control $u$ represents the cooling flow rate in a jacket around the reactor. The simulation results are shown in figure 2 , where the curves of state and control variables of $\mathrm{k}=1,2,3,4$ are left out in order to show the other curves more clearly. Performance index values at different iteration steps are listed in table 1 .

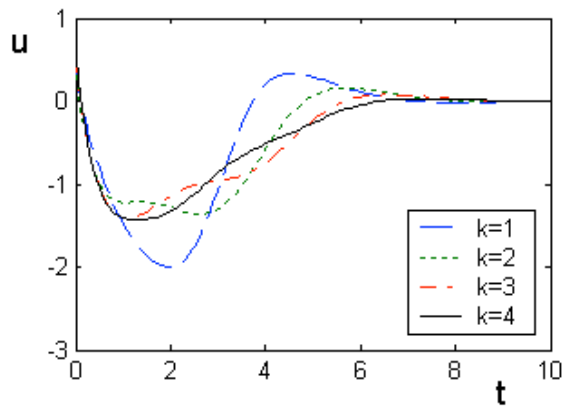

Figure 2. Curves for control law when $k=1,2,3,4$

TABLE I.

\begin{tabular}{cccccc}
\hline$k$ & & 1 & 2 & 3 & 4 \\
\hline$J_{k}$ & & 1.45 & 1.0712 & 1.0030 & 0.9998 \\
& & & & \\
\hline
\end{tabular}

According to figure 2 and table 1, it is clear that the more iterative steps, the higher the control precision. If we choose $\varepsilon=0.005$, then the relative error of the performance index values satisfies $\left|\left(J_{4}-J_{3}\right) / J_{4}\right|<\varepsilon$. 
PAPER

CONGESTION CONTROL FOR NONLINEAR TD-SCDMA DiSCRETE NETWORKS BASED ON TCP/IP

It indicates that the suboptimal control law $u_{4}$ is very close to the optimal control law $u *$. It is important to notice that in the proposed algorithm only a few iteration steps are required in order to get the suboptimal control law.

\section{CONCLUSIONS}

In this paper, the decision-making law has been studied for TD-SCDMA networked control systems based on TCP/IP. The presented approach only requires solving a sequence of linear vector differential equations instead of a sequence of the Riccati matrix differential equations or a sequence of Lyapunov matrix differential equations. A suboptimal control law can be obtained by using the finite-time approximation of the nonlinear compensation term of the optimal solution sequence. Simulation results show that the proposed approach is effective and easily implemented.

\section{ACKNOWLEDGMENT}

This work is supported by the science and technology research project of Chongqing City Board of Education (KJ1402909); the "twelfth five-year" project of Chongqing municipal education science (2014-GX-055).

\section{REFERENCES}

[1] Y. Huaicheng, et al, "Delay-dependent robust stability of uncertain networked control systems with multiple state time-delay," Journal of Control Theory and Applications, vol.11, pp.164-170, November 2007.

[2] J. Nilsson, B. Bernhardsson, and B. Wittenmark, "Stochastic analysis and control of real-time systems with random time delays," Automatica, vol.34, pp57-64, October 1998. http://dx.doi.org/10.1016/S0005-1098(97)00170-2

[3] P. Liu, "A New Congestion Controller for Multilayer Networked Control Systems with persistent Disturbances," Key Engineering
Materials, vol.2, pp.805-810, October 2010 http://dx.doi.org/10.4028/www.scientific.net/KEM.439-440.805

[4] C. J. Sreenan, U. Roedig, J. Brown, et al, "Performance Control in Wireless Sensor Networks," in Proceeding of the 6th Annual IEEE Communications Society Conference on Sensor, Mesh and Ad Hoc Communications and Networks Workshop, August 2009. pp.1-3. http://dx.doi.org/10.1109/sahenw.2009.5172953

[5] E. Munivel, G. M. Ajit, "Efficient Public Key Infrastructure Implementation in Wireless Sensor Networks, " in Proceeding of 2010 International Conference on Wireless Communication and Sensor Computing, August 2010, pp.1-6. http://dx.doi.org/10.1109/icwcsc.2010.5415904

[6] A. Ferreira, A. Goldman, J. Monteiro, "Performance Evaluation of Routing Protocols for MANETs with Known Connectivity Patterns Using Evolving Graphs," Wireless Networks, vol.16, pp.627-640, March 2010. http://dx.doi.org/10.1007/s11276-008$\underline{0158-6}$

[7] W. Wu, Z. Z. Zhang, D.Y. Qin, C.G. He, "Mitigating Congestion in Wireless Ad Hoc Networks by using a Potential-based Routing Algorithm, " in Proceeding of International Conference on Space Information Technology, May 2009, pp.501-507. http://dx.doi.org/10.1117/12.855173

[8] S. Panichpapiboon, G. Ferrari, O. K. Tonguz, "Connectivity of Ad Hoc Wireless Networks; An Alternative to Graph-Theoretic Approaches," Wireless Networks, vol.16, pp. 793-811, March 2010. http://dx.doi.org/10.1007/s11276-009-0169-y

[9] P. Liu, "A New Decision-Making for Nonlinear Input-Output Systems with Persistent Disturbances, " in Proceeding of 2010 International Conference on the Development of Educational Science and Computer Technology, January 2010, pp.422-425.

\section{AUTHORS}

Peng Liu is with Chongqing College of Electronic Engineering, China (e-mail: pengliu789@126.com).

This work is supported by the science and technology research project of Chongqing City Board of Education (KJ1402909); the "twelfth fiveyear" project of Chongqing municipal education science (2014-GX-055). Submitted, January, 17, 2015. Published as resubmitted by the authors on May, 16,2015. 